\title{
Potential Use of Spectral Reflectance Indices as a Selection Tool for Grain Yield in Winter Wheat under Great Plains Conditions
}

\author{
B. Prasad, B. F. Carver, M. L. Stone, M. A. Babar, W. R. Raun, and A. R. Klatt`
}

\begin{abstract}
Selection criteria that would facilitate increased genetic gain for grain yield would be considered advantageous in plant breeding programs. We evaluated the potential of spectral reflectance indices (SRI) for assessing grain yield variability in winter wheat (Triticum aestivum L.) genotypes under Great Plains conditions. One experiment was conducted at two locations on the Oklahoma State University research farms for $1 \mathrm{yr}$, and two experiments were conducted for $2 \mathrm{yr}$ at a single location. The first experiment included 25 winter wheat cultivars from the Great Plains, and the other two experiments contained two groups of $25 \mathrm{~F}_{4: 6}$ and $\mathrm{F}_{4: 7}$ recombinant inbred lines. Six reported SRI (red and green normalized difference vegetation index, RNDVI, GNDVI; simple ratio, SR; water index, Wl; normalized water indices 1 and 2, NWI-1 and NWI-2), and two new normalized water indices (NWI-3 and NWI-4) were calculated at booting, heading, and early grain-filling stages using a FieldSpec UV/VNIR spectroradiometer. Significant genotypic variation was observed for SRI and growth stages, though the booting stage was least associated with grain yield. The relationships of grain yield with $\mathrm{WI}$ and NWI were stronger than with the RNDVI and SR. The WI and the NWI performed better in identifying superior genotypes, either at any individual growth stage or in a combination of growth stages. Our study clearly demonstrated the potential of using SRI as a selection tool for grain yield in a winter wheat breeding program.
\end{abstract}

B. Prasad, B.F. Carver, W.R. Raun, and A.R. Klatt, Dep. of Plant and Soil Sciences, 368 Ag Hall, Oklahoma State Univ., Stillwater, OK 74078; M.L. Stone, Dep. of Biosystems and Agricultural Engineering, Oklahoma State Univ., Stillwater, OK 74078; M.A. Babar, Dep. of Agronomy, Kansas State Univ., Manhattan, KS 66506. This research was partially funded by Oklahoma Agricultural Experiment Station, Oklahoma Wheat Commission, and Oklahoma Wheat Research Foundation. Received 25 July 2006. *Corresponding author (art.klatt@okstate.edu).

Abbreviations: GNDVI, green normalized difference vegetation index; LAI, leaf area index; NDVI, normalized difference vegetation index; NIR, near infrared; NWI, normalized water indices; NWI-1, normalized water index 1; NWI-2, normalized water index 2; NWI-3, normalized water index 3; NWI-4, normalized water index 4; PAR, photosynthetically active radiation; RIL, recombinant inbred lines; RNDVI, red normalized difference vegetation index; SR, simple ratio; SRI, spectral reflectance indices; WI, water index.

$\mathrm{B}$ REEDING STRATEgies for developing higher-yielding genotypes of wheat (Triticum aestivum L.) are extensively based on approaches that involve generating a large number of crosses and subsequently selecting among and within segregating populations. To date, wheat breeding around the world has been based primarily on empirical selection criteria (yield per se) for yield improvement (Araus et al., 2002). Since yield is characterized by low heritability and a high genotype-environment interaction, empirical selection may be insufficient for achieving genetic gain (Jackson et al., 1996).

Identifying promising genotypes in a breeding program will be facilitated if grain yield can be predicted before the crop is harvested. This early prediction would also be very helpful if the topperforming families could be identified from a group of hundreds

Published in Crop Sci. 47:1426-1440 (2007).

doi: 10.2135/cropsci2006.07.0492

(C) Crop Science Society of America

677 S. Segoe Rd., Madison, WI 53711 USA

All rights reserved. No part of this periodical may be reproduced or transmitted in any form or by any means, electronic or mechanical, including photocopying, recording, or any information storage and retrieval system, without permission in writing from the publisher. Permission for printing and for reprinting the material contained herein has been obtained by the publisher. 
or thousands of segregating populations in a breeding program (Royo et al., 2003). Reynolds et al. (1999) indicated that morphological characters such as number of grains per unit area and harvest index can be used in the visual selection of breeding lines, but those traits are difficult to measure in a large number of small plots in early generations due to labor intensiveness and time.

Yield in a given environment is directly and indirectly influenced by morphological, physiological, and environmental factors. Selection of breeding lines for grain yield in advanced nurseries often needs repetition to make a selection decision because commonly used statistical procedures sometimes fail to produce sufficiently accurate results for identifying superior genotypes (Ball and Konzak, 1993). An analytical breeding strategy is an alternative breeding approach that requires a better understanding of the factors responsible for development, growth, and yield (Richards, 1982). This strategy considers morphophysiological selection criteria that have potential to make empirical selection more efficient (Reynolds et al., 2001). The limited application of this analytical approach is probably due to the lack of an appropriate understanding of the physiological parameters, their estimation, and their true associations with grain yield (Richards, 1996). Commonly used physiological selection criteria include stomatal conductance, canopy temperature depression, and $\mathrm{C}_{13}$ isotope discrimination of grains (Reynolds et al., 1999). Spectral properties of the plant came into focus as a potential selection tool for grain yield in more recent years (Aparicio et al., 2002; Royo et al., 2003; Babar et al., 2006).

The basic principle governing canopy spectral reflectance is that specific plant traits are associated with the absorption of specific wavelengths of the spectrum (Reynolds et al., 1999). For example, leaf pigments like chlorophyll, xanthophylls, and carotenoids strongly absorb light in the photosynthetically active radiation (PAR) portion of the spectrum, but not in the near infrared (NIR) region. These pigments reduce reflectance of PAR, but not NIR where the magnitude of reflectance is influenced by the scattering and diffusion of light by different leaf tissues (Knipling, 1970). Spectral reflectance by a crop canopy is related to the overall area of leaves and other photosynthetic organs in the canopy, pigment concentration, and other physiological factors (Araus et al., 2001). Thus, the measurement of the spectrum reflected from plants provides information that can be used to estimate a large number of parameters (Araus et al., 2001), such as green biomass of the canopy, photosynthetic area, amount of PAR absorbed by the canopy, and photosynthetic potential (Reynolds et al., 2001). Other parameters related to the physiological status of the canopy at the time of measurement, such as relative water content, nutrient deficiencies, environmental stresses, pigment concentration, and photosynthetic radiation use efficiency, have been assessed by spectral reflec- tance measurements (Araus et al., 2001; Reynolds et al., 2001). Several researchers have suggested that grain yield can be estimated using spectral reflectance during different crop growth stages (Araus et al., 2001; Aparicio et al., 2002; Osborne et al., 2002; Babar et al., 2006).

The most commonly used spectral reflectance indices (SRI) are simple ratio (SR) and normalized difference vegetative index (NDVI) (Araus et al., 2002). Green biomass, leaf area index (LAI), green area index, green leaf area index, and fraction of absorbed photosynthetically active radiation are positively correlated with SRI (Wiegand and Richardson, 1990a, 1990b; Baret and Guyot, 1991; Price and Bausch, 1995). Measuring SRI periodically during different crop growth stages allows the estimation of leaf area duration that serves as an indicator of stress tolerance and the total PAR absorbed by the canopy, which are the most important factors for predicting yield (Wiegand and Richardson, 1990a). Stress assessment in plants is one of the important physiological tools associated with certain spectral indices. Water index (WI) has been demonstrated to predict relative water content, leaf water potential, stomatal conductance, and canopy temperature with sufficient water stress (Peñuelas et al., 1993). Peñuelas et al. (1997) showed the usefulness of using WI to assess the effect of salinity on barley.

Experiments in multiple locations for predicting grain yield of wheat using SRI have shown that approximately 50 to $65 \%$ of yield variability can be explained by NDVI and SR (Tucker et al., 1980; Aparicio et al., 2000; Serrano et al., 2000; Raun et al., 2001). At LAI values above 3, the canopy absorbs all of the incident red light, leaving no more red light to be absorbed by higher vegetation fractions. Hence, the common SRI that utilize the red band are insensitive to variation in vegetation fraction or LAI values beyond 3 (Serrano et al., 2000). Therefore, these SRI cannot be used as a selection tool in wheat breeding programs where genotypes are selected at later reproductive growth stages.

This study explored the spectrum for wavelengths or combinations of wavelengths that could be used to adequately predict winter wheat genotypes for grain yield variation. Specific objectives were to test the correlation of widely used SRI with grain yield variability, to identify new spectral indices better correlated with grain yield variability among winter wheat genotypes across environments, and to determine the optimum growth stage(s) for measurement of SRI.

\section{MATERIALS AND METHODS Experimental Materials}

Three experiments, each containing 25 winter wheat genotypes developed by different breeding programs in the southern and central Great Plains, were conducted during the 2003/2004 and 2004/2005 wheat growing seasons. The experiments were 
Table 1. Mean, maximum, and minimum monthly temperatures $\left({ }^{\circ} \mathrm{C}\right)$ and monthly total rainfall $(\mathrm{mm})$ for the two growing seasons at two locations.

\begin{tabular}{|c|c|c|c|c|c|c|c|c|c|c|}
\hline Parameters & & Oct. & Nov. & Dec. & Jan. & Feb. & Mar. & Apr. & May & Mean/sum ${ }^{\dagger}$ \\
\hline \multicolumn{11}{|c|}{ Stillwater $(2003 / 2004)$} \\
\hline \multirow[t]{3}{*}{ Mean temp. $\left({ }^{\circ} \mathrm{C}\right)$} & Max. & 23.9 & 15.0 & 11.7 & 9.4 & 10.0 & 18.3 & 21.7 & 28.3 & 17.3 \\
\hline & Min. & 8.9 & 3.9 & -0.6 & -2.8 & -2.2 & 5.6 & 8.9 & 15.6 & 4.7 \\
\hline & Ave. & 16.3 & 9.7 & 5.5 & 3.2 & 3.7 & 12.4 & 15.6 & 22.0 & 11.1 \\
\hline Total rainfall (mm) & & 74.9 & 54.4 & 42.7 & 56.9 & 42.4 & 101.1 & 71.4 & 5.8 & 449.6 \\
\hline \multicolumn{11}{|c|}{ Stillwater (2004/2005) } \\
\hline \multirow[t]{3}{*}{ Mean temp. $\left({ }^{\circ} \mathrm{C}\right)$} & Max. & 23.3 & 15.0 & 12.8 & 8.9 & 13.3 & 16.7 & 22.8 & 26.7 & 17.4 \\
\hline & Min. & 11.1 & 5.0 & -2.2 & -2.2 & 1.1 & 3.3 & 8.9 & 14.4 & 4.9 \\
\hline & Ave. & 17.1 & 9.8 & 4.9 & 2.6 & 6.9 & 10.3 & 15.8 & 20.4 & 11.0 \\
\hline Total rainfall $(\mathrm{mm})$ & & 116.1 & 126.0 & 24.4 & 70.4 & 33.3 & 17.5 & 9.9 & 97.8 & 495.4 \\
\hline \multicolumn{11}{|c|}{ Lake Carl Blackwell (2003/2004) } \\
\hline \multirow[t]{3}{*}{ Mean temp. $\left({ }^{\circ} \mathrm{C}\right)$} & Max. & 23.9 & 15.0 & 11.7 & 9.4 & 10.0 & 18.3 & 21.7 & 27.8 & 17.2 \\
\hline & Min. & 10.0 & 4.4 & 0.0 & -2.8 & -2.2 & 6.1 & 10.0 & 15.0 & 5.1 \\
\hline & Ave. & 16.6 & 9.6 & 5.5 & 3.1 & 3.7 & 12.4 & 15.5 & 21.6 & 11.0 \\
\hline Total rainfall $(\mathrm{mm})$ & & 84.3 & 60.7 & 33.0 & 62.2 & 39.6 & 110.2 & 74.9 & 3.6 & 468.5 \\
\hline \multicolumn{11}{|c|}{ Lake Carl Blackwell (2004/2005) } \\
\hline \multirow[t]{3}{*}{ Mean temp. $\left({ }^{\circ} \mathrm{C}\right)$} & Max. & 23.3 & 14.4 & 12.8 & 8.3 & 12.8 & 16.7 & 22.2 & 26.1 & 17.1 \\
\hline & Min. & 11.1 & 5.0 & -1.1 & -2.8 & 1.7 & 3.9 & 9.4 & 14.4 & 5.2 \\
\hline & Ave. & 17.1 & 9.7 & 5.2 & 2.6 & 7.2 & 10.2 & 15.7 & 19.9 & 11.0 \\
\hline Total rainfall (mm) & & 127.8 & 162.6 & 24.1 & 65.3 & 38.4 & 19.6 & 10.7 & 69.1 & 517.6 \\
\hline Long-term ${ }^{\ddagger}$ average temp. $\left({ }^{\circ} \mathrm{C}\right)$ & & 16.0 & 8.2 & 2.5 & 1.2 & 3.6 & 5.9 & 14.3 & 19.7 & 8.9 \\
\hline Total rainfall $(\mathrm{mm})$ & & 59.9 & 41.9 & 29.2 & 22.4 & 29.5 & 47.5 & 71.1 & 105.6 & 407.1 \\
\hline
\end{tabular}

tValues represent mean temperature and total rainfall from Oct. to May.

*Data represent both sites averaged over past $100 \mathrm{yr}$.

planted at the Oklahoma State University Agronomy Research Farm at Stillwater, Oklahoma $\left(36^{\circ} 11^{\prime} \mathrm{N}, 9^{\circ} 05^{\prime} \mathrm{W}\right)$, and at Lake Carl Blackwell $\left(36^{\circ} 16^{\prime} \mathrm{N}, 9^{\circ} 28^{\prime} \mathrm{W}\right)$, located $25 \mathrm{~km}$ west of Stillwater. The soil types at these sites are Kirkland silt loam (fine, mixed, thermic Udertic Paleustolls) with an average $\mathrm{pH}$ of 6.2 to 6.5 , and Pulaski fine sandy loam (coarse-loamy, mixed, nonacid, thermic Typic Ustifluvent) with an average $\mathrm{pH}$ of 6.7 to 6.9. The weather patterns of the two sites are presented in Table 1. All four site-years were above the long-term average temperature $\left(8.9^{\circ} \mathrm{C}\right)$ and long-term total precipitation $(407.1$ $\mathrm{mm}$ ), although March and April were drier in 2004/2005 at both sites compared to the long-term average.

The first experiment, designated as Exp-1, was composed of commercial winter wheat cultivars from the southern and central Great Plains and was planted at both sites in year 2003/2004. The second experiment, designated as Exp-2, was composed of $25 \mathrm{~F}_{4 \cdot 6}$ and $\mathrm{F}_{4.7}$ recombinant inbred lines (RIL) from the cross TX 95V5905/Jagger and was planted at the Lake Carl Blackwell site in years 2003/2004 ( $\left.\mathrm{F}_{6}\right)$ and 2004/2005 $\left(\mathrm{F}_{7}\right)$. These lines were developed by the winter wheat breeding program at Kansas State University, Manhattan. The third experiment, designated as Exp-3, was composed of $25 \mathrm{~F}_{4: 6}$ and $\mathrm{F}_{4: 7}$ RIL from the cross TX93V4927/G1878 and was planted at the Stillwater site in years 2003/2004 $\left(\mathrm{F}_{6}\right)$ and 2004/2005 $\left(\mathrm{F}_{7}\right)$. This population was developed by AgriPro Coker (Vernon, TX).

All experiments were conducted under rain-fed conditions and planted at a seeding rate of $70 \mathrm{~kg} \mathrm{ha}^{-1}$. Individual plot size for each experiment was $3 \mathrm{~m}$ long $\times 1.2 \mathrm{~m}$ wide. Each individual experiment was a $5 \times 5$ alpha lattice design with two replica- tions. At both experimental sites, $90 \mathrm{~kg} \mathrm{ha}^{-1}$ preplant nitrogen was applied. Folicur 3.6F (38.7\% tebuconazole; Bayer CropScience, NC) was applied twice (late tillering, Zadoks stage 29 and booting, Zadoks stage 45 [Zadoks et al., 1974]) to control foliar diseases, and Cygon 2E (23\% dimethoate; Southern Agricultural Insecticides, Inc., FL) was applied at booting to control aphids. Grain yield for each plot was determined by mechanically harvesting the whole plot and expressed as $\mathrm{kg} \mathrm{ha}^{-1}$.

\section{Spectral Reflectance Measurements}

Spectral reflectance measurements were accomplished using a portable field spectroradiometer (FieldSpec UV/VNIR, Analytical Spectral Devices, Boulder, CO). Reflectance measurements were taken with a $25^{\circ}$ field of view optic at vertical position. The spectroradiometer was capable of measuring radiance from 350- to 1050-nm wavelengths with a sampling interval of $1.4 \mathrm{~nm}$ of the spectrum. Thus, 512 continuous data points were obtained with each reading. Measurements were taken during the middle of the day on cloudless days. The optical sensor was placed approximately $50 \mathrm{~cm}$ above the plant canopy in nadir position. The incident spectrum was taken from the light reflected from a white reference panel, and reflectance was calculated from the ratio of reflected light from the crop canopy against the total radiance reflectance from the white surface. The spectroradiometer was recalibrated every 10 plots. Four spectral measurements were taken randomly from four different places per plot, and the mean of the four readings was used to calculate the spectral reflectance indices. Reflectance data were taken at three growth stages, namely, booting, heading (Zadoks 
stage 59), and early grain-filling (Zadoks stage 75) for all three experiments in both years and locations. There was a difference of 3 to $7 \mathrm{~d}$ among the genotypes in reaching booting, heading, and early grain-filling stages. The reflectance measurements were taken at the middle of this range to ensure minimal influence on the readings. We calculated about 200 new indices using different combinations of visible and NIR wavebands in ratio and normalized forms and chose two new indices based on the overall performance across experiments and compared with six previously reported indices. Descriptions of the indices are presented in Table 2.

\section{Data Analysis}

The alpha lattice analysis was performed by SAS MIXED procedures (SAS Institute, 2001) for grain yield and spectral reflectance indices. Pearson's correlation coefficients were used to determine the association between grain yield and spectral reflectance indices measured at individual growth stages and across different growth stages. Genetic correlations between grain yield and SRI were calculated across growth stages and environments by the formula (Falconer, 1989)

$$
r_{\mathrm{g}}=\left(\operatorname{Cov}_{\mathrm{G} 1 \mathrm{G} 2}\right) / \sqrt{ }\left(\operatorname{Var}_{\mathrm{G} 1} \times \operatorname{Var}_{\mathrm{G} 2}\right)
$$

where Cov indicates components of covariance between grain yield (G1) and SRI (G2), and Var indicates components of variance of grain yield and SRI.

Relationship between SRI and grain yield was established by regression analysis and was accomplished by the SAS software (SAS Institute, 2001). Data were fitted into different models based on the least-square method of parameter estimation. Residuals were checked for model fitting. Coefficients of determination were compared for different models. Root mean square error was calculated based on work by Kobayashi and Salam (2000).

Selection for the 25\% highest-yielding and 25\% lowestyielding genotypes was done by ranking the genotypes according to grain yield and ranking the genotypes based on the SRI. Significant grain yield differences between the genotypes of the two selection groups based on the 25\% highest and the $25 \%$ lowest SRI values were tested. The genotypes were ranked for SRI based on the mean of the readings obtained by combining the reflectance data across three growth stages. We also determined the yield per se of the $25 \%$ highest-yielding and $25 \%$ lowest-yielding genotypes. The yield estimate based on individual SRI was calculated using the regression equation of that specific index with grain yield. Percent yield differences were then estimated between yield per se and yield estimates based on different SRI.

\section{RESULTS}

\section{Genotypic Variation and Growth Stage}

Significant genotypic differences $(p<0.05)$ for grain yield were found in all three experiments (Table 3 ). Differences $(p<0.05)$ were observed for spectral behavior at different crop growth stages, namely booting, heading, and early grain-filling (Table 4). The values for RNDVI, GNDVI, and SR decreased from the booting to the grain-filling stage (Table 4, Fig. 1). On the other hand WI, NWI-1, NWI-2, NWI-3, and NWI-4 provided two different types of patterns (Fig. 1). In year 2003/2004, these indices

Table 2. Description of the spectral reflectance indices employed in this study.

\begin{tabular}{llll}
\hline \multicolumn{1}{c}{ Spectral reflectance indices } & \multicolumn{1}{c}{ Estimation ${ }^{\dagger}$} & Function & References \\
\hline Red normalized difference vegetation index (RNDVI) & $\left(R_{780}-R_{670}\right) /\left(R_{780}+R_{670}\right)$ & Canopy photosynthetic area & Raun et al., 2001 \\
Green normalized difference vegetation index (GNDVI) & $\left(R_{780}-R_{550}\right) /\left(R_{780}+R_{550}\right)$ & Canopy photosynthetic area & Aparicio et al., 2000 \\
Simple ratio (SR) & $\left(R_{900} / R_{680}\right)$ & Canopy photosynthetic area & Gitelson et al., 1996 \\
Water index (WI) & $\left(R_{970} / R_{900}\right)$ & Canopy water status & Peñuelas et al., 1993 \\
Normalized water index 1 (NWI-1) & $\left(R_{970}-R_{900}\right) /\left(R_{970}+R_{900}\right)$ & Canopy water status & Babar et al., 2006 \\
Normalized water index 2 (NWI-2) & $\left(R_{970}-R_{850}\right) /\left(R_{970}+R_{850}\right)$ & Canopy water status & Babar et al., 2006 \\
Normalized water index 3 (NWI-3) & $\left(R_{970}-R_{920}\right) /\left(R_{970}+R_{920}\right)$ & Canopy water status & Newly developed \\
Normalized water index 4 (NWI-4) & $\left(R_{970}-R_{880}\right) /\left(R_{970}+R_{880}\right)$ & Canopy water status & Newly developed \\
\hline
\end{tabular}

${ }^{+} R$, reflectance at a specific wavelength of the light spectrum (in $\mathrm{nm}$ ).

Table 3. Statistical parameters for grain yield in three experiments presented as individual year/location.

\begin{tabular}{|c|c|c|c|c|c|c|c|}
\hline \multirow{2}{*}{\multicolumn{2}{|c|}{ Parameters }} & \multicolumn{2}{|c|}{ Exp-1 } & \multicolumn{2}{|c|}{ Exp-2 } & \multicolumn{2}{|c|}{ Exp-3 } \\
\hline & & \multirow{2}{*}{$\frac{\text { STW 03/04 }}{3920}$} & \multirow{2}{*}{$\frac{\text { LCB 03/04 }}{4229}$} & \multirow{2}{*}{$\frac{\text { LCB 03/04 }}{3566}$} & \multirow{2}{*}{$\frac{\text { LCB 04/05 }}{2482}$} & \multirow{2}{*}{$\frac{\text { STW 03/04 }}{3294}$} & \multirow{2}{*}{$\frac{\text { STW 04/05 }}{3381}$} \\
\hline Yield (kg/ha) & Min. & & & & & & \\
\hline & Max. & 6460 & 6620 & 6576 & 3512 & 5808 & 5498 \\
\hline & Mean & 4950 & 5383 & 4989 & 2980 & 4667 & 4271 \\
\hline \multicolumn{2}{|l|}{ SE } & 530 & 430 & 589 & 249 & 437 & 361 \\
\hline \multicolumn{2}{|l|}{ LSD (5\%) } & 1125 & 912 & 1250 & 527 & 927 & 765 \\
\hline \multicolumn{2}{|c|}{ CV (\%) } & 10.2 & 7.8 & 11.7 & 7.9 & 8.6 & 8.5 \\
\hline \multicolumn{2}{|c|}{ Significance level } & * & $\star *$ & * & * & $\star *$ & $\star \star$ \\
\hline
\end{tabular}

*Significant at the 0.05 probability level.

**Significant at the 0.01 probability level.

+STW, Stillwater site; LCB, Lake Carl Blackwell site. 
Table 4. Statistical parameters of different spectral reflectance indices estimated at different growth stages, presented for three experiments across different years and locations.

\begin{tabular}{|c|c|c|c|c|c|c|c|c|c|c|}
\hline \multirow{2}{*}{ Indices $^{\dagger}$} & \multirow{2}{*}{ Parameters } & \multicolumn{3}{|c|}{ Exp-1 $1^{\ddagger}$} & \multicolumn{3}{|c|}{ Exp-2§ } & \multicolumn{3}{|c|}{ Exp-3§ } \\
\hline & & Booting & Heading & Grain-filling & Booting & Heading & Grain-filling & Booting & Heading & Grain-filling \\
\hline \multirow[t]{5}{*}{ WI } & Minimum & 0.810 & 0.784 & 0.891 & 0.824 & 0.843 & 0.866 & 0.806 & 0.794 & 0.869 \\
\hline & Maximum & 0.876 & 0.906 & 0.964 & 0.866 & 0.910 & 0.980 & 0.871 & 0.870 & 0.953 \\
\hline & Mean & 0.842 & 0.849 & 0.935 & 0.847 & 0.865 & 0.915 & 0.835 & 0.833 & 0.903 \\
\hline & SE & 0.012 & 0.013 & 0.015 & 0.015 & 0.013 & 0.012 & 0.008 & 0.013 & 0.015 \\
\hline & Significance level & 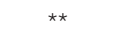 & ** & $\star \star$ & * & & $\star \star$ & $\star \star$ & $\star \star$ & $\star \star$ \\
\hline \multirow[t]{5}{*}{ RNDVI } & Minimum & 0.928 & 0.779 & 0.524 & 0.841 & 0.757 & 0.518 & 0.886 & 0.869 & 0.612 \\
\hline & Maximum & 0.945 & 0.943 & 0.834 & 0.941 & 0.900 & 0.828 & 0.948 & 0.933 & 0.837 \\
\hline & Mean & 0.870 & 0.887 & 0.651 & 0.900 & 0.851 & 0.680 & 0.931 & 0.904 & 0.724 \\
\hline & SE & 0.016 & 0.028 & 0.046 & 0.018 & 0.028 & 0.036 & 0.009 & 0.016 & 0.051 \\
\hline & Significance level & & * & $\star \star *$ & * & & $\star \star$ & $\star \star$ & $\star \star$ & $\star \star$ \\
\hline \multirow[t]{5}{*}{ GNDVI } & Minimum & 0.755 & 0.660 & 0.564 & 0.768 & 0.680 & 0.620 & 0.773 & 0.776 & 0.606 \\
\hline & Maximum & 0.846 & 0.858 & 0.736 & 0.830 & 0.810 & 0.741 & 0.869 & 0.849 & 0.746 \\
\hline & Mean & 0.808 & 0.780 & 0.633 & 0.799 & 0.757 & 0.668 & 0.835 & 0.811 & 0.672 \\
\hline & SE & 0.018 & 0.023 & 0.026 & 0.016 & 0.020 & 0.021 & 0.01 & 0.015 & 0.027 \\
\hline & Significance level & $\star \star$ & $\star \star$ & $\star \star$ & & * & * & $\star *$ & $\star \star$ & $\star \star$ \\
\hline \multirow[t]{5}{*}{ SR } & Minimum & 18.2 & 8.5 & 3.15 & 11.45 & 6.54 & 2.83 & 17.07 & 14.65 & 4.15 \\
\hline & Maximum & 34.9 & 33.1 & 11.22 & 32.35 & 21.82 & 9.49 & 38.59 & 28.04 & 11.36 \\
\hline & Mean & 27.8 & 19.9 & 5.12 & 21.49 & 13.44 & 5.52 & 29.07 & 20.49 & 6.81 \\
\hline & SE & 3.86 & 2.665 & 1.013 & 4.043 & 2.48 & 0.911 & 3.417 & 2.91 & 1.42 \\
\hline & Significance level & * & $\star \star$ & $\star \star$ & & & * & $\star \star$ & $\star \star$ & $\star \star$ \\
\hline \multirow[t]{5}{*}{ NWI-1 } & Minimum & -0.105 & -0.121 & -0.058 & -0.096 & -0.085 & -0.072 & -0.108 & -0.115 & -0.070 \\
\hline & Maximum & -0.067 & -0.049 & -0.019 & -0.072 & -0.047 & -0.010 & -0.069 & -0.07 & -0.024 \\
\hline & Mean & -0.086 & -0.081 & -0.034 & -0.083 & -0.072 & -0.044 & -0.09 & -0.09 & -0.051 \\
\hline & SE & 0.007 & 0.007 & 0.008 & 0.009 & 0.008 & 0.007 & 0.005 & 0.008 & 0.008 \\
\hline & Significance level & $\star \star *$ & ** & 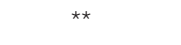 & * & $\star *$ & 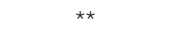 & $\star \star *$ & 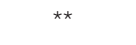 & 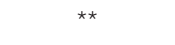 \\
\hline \multirow[t]{5}{*}{ NWI-2 } & Minimum & -0.107 & -0.118 & -0.040 & -0.095 & -0.079 & -0.061 & -0.105 & -0.105 & -0.056 \\
\hline & Maximum & -0.066 & -0.027 & 0.015 & -0.066 & -0.022 & 0.035 & -0.053 & -0.056 & 0.007 \\
\hline & Mean & -0.084 & -0.072 & -0.005 & -0.081 & -0.063 & -0.02 & -0.087 & -0.080 & -0.032 \\
\hline & SE & 0.008 & 0.009 & 0.011 & 0.010 & 0.01 & 0.009 & 0.006 & 0.009 & 0.011 \\
\hline & Significance level & $\star \star *$ & 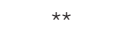 & $\star \star$ & * & * & * & $\star \star *$ & ** & ** \\
\hline \multirow[t]{5}{*}{ NWI-3 } & Minimum & -0.102 & -0.118 & -0.061 & -0.094 & -0.084 & -0.072 & -0.105 & -0.111 & -0.071 \\
\hline & Maximum & -0.067 & -0.054 & -0.026 & -0.071 & -0.052 & -0.022 & -0.070 & -0.070 & -0.031 \\
\hline & Mean & -0.084 & -0.082 & -0.041 & -0.082 & -0.073 & -0.049 & -0.08 & -0.089 & -0.054 \\
\hline & SE & 0.006 & 0.007 & 0.007 & 0.008 & 0.007 & 0.006 & 0.004 & 0.007 & 0.007 \\
\hline & Significance level & $\star *$ & $\star \star *$ & $\star * *$ & * & & ** & $\star \star *$ & ** & $* *$ \\
\hline \multirow[t]{5}{*}{ NWI-4 } & Minimum & -0.017 & -0.125 & -0.055 & -0.101 & -0.087 & -0.073 & -0.111 & -0.113 & -0.070 \\
\hline & Maximum & -0.068 & -0.043 & -0.010 & -0.074 & -0.041 & 0.004 & -0.066 & -0.067 & -0.015 \\
\hline & Mean & -0.087 & -0.082 & -0.027 & -0.086 & -0.073 & -0.040 & -0.092 & -0.090 & -0.048 \\
\hline & SE & 0.008 & 0.008 & 0.009 & 0.01 & 0.008 & 0.008 & 0.005 & 0.008 & 0.009 \\
\hline & Significance level & $\star \star$ & $\star \star$ & $\star *$ & * & * & $\star *$ & $\star *$ & $\star \star$ & $\star \star$ \\
\hline
\end{tabular}

*Significant at the 0.05 probability level.

**Significant at the 0.01 probability level.

'GNDVI, green normalized difference vegetation index; NWI-1, normalized water index 1; NWI-2, normalized water index 2; NWI-3, normalized water index 3; NWI-4, normalized water index 4; RNDVI, red normalized difference vegetation index; SR, simple ratio; WI, water index.

EEstimates based on two locations in year 2003/2004.

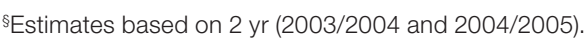

decreased from booting to heading, and then increased at the grain-filling stage (Fig. 1a, b), but in 2004/2005, they showed a continuous increase from the booting to the grain-filling stage (Fig. 1c, d). In all instances, the values of the water-based indices were higher in the grain-filling stage compared to booting stage (Table 4, Fig. 1).

\section{Interaction between Genotypes, Growth Stages, and Years/Locations}

The ANOVA for Exp-3 (Table 5) revealed that genotypes and growth stage main effects were significant, as well as the growth stage by genotype interaction. The three-way interactions were significant for all the water-based NIR 

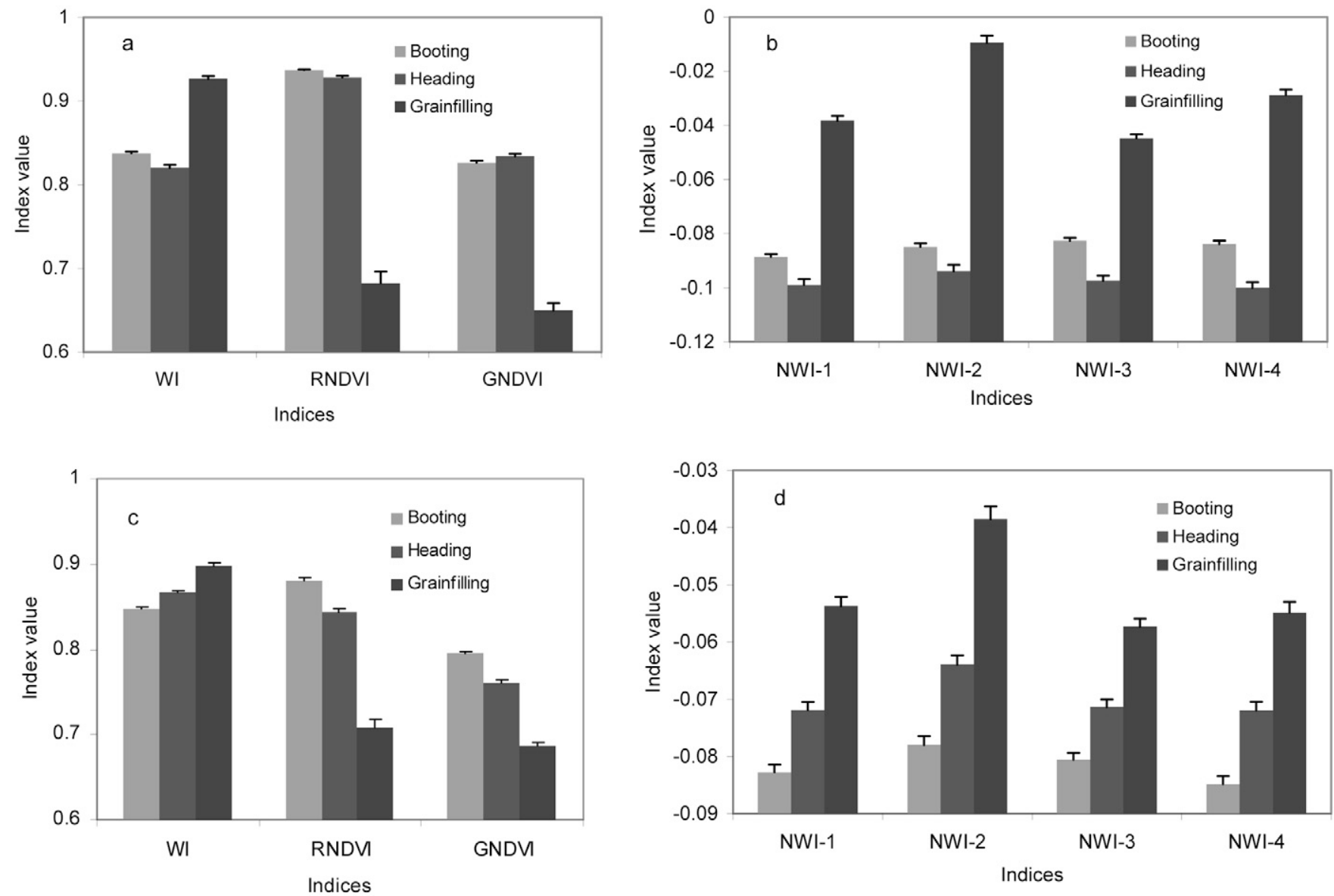

Figure 1. Changes in the pattern of different spectral reflectance indices with the advancement of growth stages: (a) and (b) represent Exp-1 in year 2003/2004 at Stillwater, and (c) and (d) represent Exp-2 in 2004/2005 at Lake Carl Blackwell. GNDVI, green normalized difference vegetation index; NWI-1, normalized water index 1; NWI-2, normalized water index 2; NWI-3, normalized water index 3; NWI-4, normalized water index 4; RNDVI, red normalized difference vegetation index; WI, water index. The bars indicate standard errors.

indices. The analyses of variance for the other two experiments were similar (data not presented). The correlation coefficients between the heading and grain-filling stages for each individual index value were significant for most experiments across locations and years (Table 6). On the other hand, the correlation coefficients of the index value between booting and heading stages, as well as between the booting and grain-filling stages, were nonsignificant in most cases (Table 6).

\section{Correlation between Spectral Reflectance Indices and Grain Yield}

Among the tested indices, six have been previously studied (WI, RNDVI, GNDVI, SR, NWI-1, and NWI-2), and two new indices (NWI-3 and NWI-4) have been calculated. The reported indices have been previously used to estimate different physiological parameters and yield, mostly with durum wheat (Triticum turgidum L. subsp. durum) under Mediterranean rain-fed conditions or spring wheat (Triticum aestivum L.) under irrigation. Three indices (RNDVI, GNDVI, and SR) provided positive associations with grain yield, whereas, the other five showed negative associations (Table 7). Almost all the indices showed low correlation with grain yield at the booting stage and high correlations at heading and grain-filling stages with grain yield. The indices can be classified into two groups. One group includes the visible and NIR wavebands, and another group includes only the NIR wavebands, especially the minor water absorption band of $970 \mathrm{~nm}$ (Table 2). The indices based on the minor water absorption band tended to provide higher correlations with grain yield compared to the widely used RNDVI, GNDVI, and SR. In each individual experiment, the NIR-based indices exhibited a consistent negative association with grain yield across years and locations. The vegetation-based indices (RNDVI, GNDVI, and SR) gave correlations that were inconsistent in different experiments across years and locations.

Mean indices over the three growth stages always provided higher correlations with grain yield compared to any individual growth stage (Table 7), except for the RNDVI, GNDVI, and SR indices in certain cases. The indices based on the minor water absorption band always provided a higher association with grain yield compared to the RNDVI, GNDVI, and SR when the mean of the three growth stages was used. These patterns were also evident when indices were combined for the heading and grain-filling stages, but 
Table 5. Combined analysis of variance (mean squares) for different spectral reflectance indices across different years and growth stages (GS) in Exp-3.

\begin{tabular}{|c|c|c|c|c|c|c|c|c|c|}
\hline \multicolumn{10}{|c|}{ Spectral reflectance indices ${ }^{\dagger}$} \\
\hline Source of variation & df & WI & RNDVI & GNDVI & SR & NWI-1 & NWI-2 & NWI-3 & NWI-4 \\
\hline Year & 1 & 0.00091 & 0.000744 & 0.015693 & 7.88 & 0.000319 & 0.000109 & 0.000247 & 0.000438 \\
\hline Rep (year) & 2 & $0.00523^{\star \star}$ & $0.02528^{\star \star}$ & $0.00965^{\star \star}$ & $395.84^{\star \star}$ & $0.00175^{\star \star}$ & $0.002919^{\star \star}$ & $0.00149^{\star *}$ & $0.002424^{* \star}$ \\
\hline Block (year × rep) & 16 & 0.00036 & 0.001430 & 0.000548 & $21.63^{\star \star}$ & 0.000106 & $0.000187^{\star}$ & $0.000092^{*}$ & 0.000154 * \\
\hline Genotype & 24 & $0.00175^{\star \star}$ & $0.006872^{* \star}$ & $0.00355^{\star *}$ & $79.87^{\star \star}$ & $0.00059^{* *}$ & $0.000874^{\star \star}$ & $0.00046^{\star \star}$ & $0.000702^{\star \star}$ \\
\hline GS & 2 & $0.15693^{\star \star}$ & $1.26649^{* *}$ & $0.77409^{\star \star}$ & $12608^{\star \star}$ & $0.05152^{\star \star}$ & $0.091245^{\star}$ & $0.04003^{\star \star}$ & $0.062720^{*}$ \\
\hline GS × genotype & 48 & $0.00049^{*}$ & $0.00322^{\star \star}$ & $0.00118^{\star \star}$ & $19.47^{\star \star}$ & $0.000167^{*}$ & $0.000248^{*}$ & $0.000135^{\star}$ & $0.000199^{*}$ \\
\hline Year $\times$ genotype & 24 & 0.00029 & 0.001126 & 0.000499 & $15.41^{\star}$ & 0.000097 & 0.000140 & 0.000073 & 0.000110 \\
\hline Year $\times$ GS & 2 & 0.00048 & 0.000281 & $0.00417^{\star \star}$ & 9.99 & 0.000141 & $0.001964^{* *}$ & 0.000055 & $0.00075^{\star \star}$ \\
\hline Year $\times$ GS $\times$ genotype & 48 & $0.00029^{\star \star}$ & 0.000916 & 0.000375 & 9.34 & $0.000095^{\star *}$ & $0.000134^{* *}$ & $0.000075^{\star *}$ & $0.00011^{\star \star}$ \\
\hline Residual & 132 & & & & & & & & \\
\hline Total & 299 & & & & & & & & \\
\hline
\end{tabular}

*Significant at the 0.05 probability level.

**Significant at the 0.01 probability level.

${ }^{\dagger}$ GNDVI, green normalized difference vegetation index; NWI-1, normalized water index 1; NWI-2, normalized water index 2; NWI-3, normalized water index 3; NWI-4, normalized water index 4; RNDVI, red normalized difference vegetation index; SR, simple ratio; WI, water index.

Table 6. Correlation coefficients between the estimates of spectral reflectance indices among different growth stages in three experiments for two locations/years.

\begin{tabular}{|c|c|c|c|c|c|c|}
\hline \multicolumn{7}{|l|}{ Indices $^{\dagger}$} \\
\hline \multirow[t]{2}{*}{ Exp-1 } & \multicolumn{3}{|c|}{ STW 03/04 } & \multicolumn{3}{|c|}{ LCB 03/04 } \\
\hline & Boot-Hd ${ }^{\ddagger}$ & Boot-Gfs & Hd-Gfף & Boot-Hd & Boot-Gf & Hd-Gf \\
\hline WI & 0.217 & 0.043 & 0.076 & 0.122 & -0.183 & $0.699^{\star *}$ \\
\hline RNDVI & 0.043 & -0.317 & 0.373 & 0.273 & -0.133 & $0.602^{\star \star}$ \\
\hline GNDVI & 0.310 & -0.135 & 0.071 & 0.389 & 0.296 & $0.667^{\star \star}$ \\
\hline $\mathrm{SR}$ & -0.003 & -0.218 & 0.324 & 0.106 & -0.035 & $0.792^{\star *}$ \\
\hline NWI-1 & 0.208 & 0.031 & 0.074 & 0.133 & -0.185 & $0.694^{\star \star}$ \\
\hline NWI-2 & 0.258 & 0.102 & 0.057 & 0.005 & -0.272 & $0.679^{\star \star}$ \\
\hline NWI-3 & 0.257 & 0.078 & 0.096 & 0.148 & -0.118 & $0.725^{\star *}$ \\
\hline $\mathrm{NWI}-4$ & 0.297 & 0.154 & 0.090 & 0.099 & -0.202 & $0.690^{\star *}$ \\
\hline \multirow[t]{2}{*}{ Exp-2 } & \multicolumn{3}{|c|}{ LCB 03/04 } & \multicolumn{3}{|c|}{ LCB 04/05 } \\
\hline & Boot-Hd & Boot-Gf & Hd-Gf & Boot-Hd & Boot-Gf & Hd-Gf \\
\hline WI & 0.289 & -0.041 & $0.664^{\star \star}$ & $0.717^{\star \star}$ & 0.367 & $0.656^{\star \star}$ \\
\hline RNDVI & 0.016 & -0.104 & $0.804^{\star *}$ & $0.690^{* \star}$ & 0.185 & $0.482^{*}$ \\
\hline GNDVI & 0.258 & 0.279 & $0.791^{\star *}$ & $0.748^{\star \star}$ & $0.415^{\star}$ & $0.604^{\star *}$ \\
\hline SR & -0.197 & -0.320 & $0.842^{\star \star}$ & $0.683^{\star \star}$ & 0.048 & 0.363 \\
\hline NWI-1 & 0.295 & -0.037 & $0.665^{\star \star}$ & $0.716^{\star \star}$ & 0.386 & $0.652^{\star \star}$ \\
\hline NWI-2 & 0.130 & -0.181 & $0.695^{\star \star}$ & $0.646^{\star \star}$ & 0.173 & $0.528^{\star \star}$ \\
\hline NWI-3 & 0.353 & -0.106 & $0.674^{\star *}$ & $0.691^{\star \star}$ & $0.459^{*}$ & $0.705^{\star *}$ \\
\hline NWI-4 & 0.205 & -0.168 & $0.666^{\star \star}$ & $0.668^{* *}$ & 0.286 & $0.593^{\star \star}$ \\
\hline \multirow[t]{2}{*}{ Exp-3 } & \multicolumn{3}{|c|}{ STW 03/04 } & \multicolumn{3}{|c|}{ STW 04/05 } \\
\hline & Boot-Hd & Boot-Gf & Hd-Gf & Boot-Hd & Boot-Gf & $\mathrm{Hd}-\mathrm{Gf}$ \\
\hline WI & $0.505^{\star \star}$ & 0.105 & $0.614^{\star *}$ & 0.126 & 0.128 & $0.804^{\star \star}$ \\
\hline RNDVI & 0.196 & -0.244 & $0.623^{\star *}$ & $0.746^{\star \star}$ & $0.489^{*}$ & $0.771^{* *}$ \\
\hline GNDVI & 0.388 & -0.119 & $0.589^{\star \star}$ & $0.707^{\star \star}$ & $0.430^{\star}$ & $0.813^{\star *}$ \\
\hline $\mathrm{SR}$ & 0.288 & -0.137 & $0.648^{\star \star}$ & $0.836^{\star \star}$ & $0.522^{\star *}$ & $0.765^{\star *}$ \\
\hline NWI-1 & $0.485^{\star}$ & 0.097 & $0.619^{\star \star}$ & 0.127 & 0.140 & $0.802^{\star \star}$ \\
\hline NWI-2 & $0.464^{*}$ & 0.081 & $0.609^{\star *}$ & 0.216 & 0.199 & $0.762^{\star *}$ \\
\hline NWI-3 & $0.506^{\star \star}$ & 0.085 & $0.605^{\star \star}$ & 0.110 & 0.140 & $0.798^{\star \star}$ \\
\hline NWI-4 & $0.425^{\star}$ & 0.107 & $0.616^{\star \star}$ & 0.173 & 0.203 & $0.770^{\star *}$ \\
\hline
\end{tabular}

*Significant at the 0.05 probability level.

**Significant at the 0.01 probability level.

${ }^{\dagger}$ GNDVI, green normalized difference vegetation index; NWI-1, normalized water index 1; NWI-2, normalized water index 2; NWI-3, normalized water index 3; NWI-4, normalized water index 4; RNDVI, red normalized difference vegetation index; SR, simple ratio; WI, water index.

‡Correlation between booting and heading stages.

${ }^{\S}$ Correlation between booting and grain-filling stages.

"Correlation between heading and grain-filling stages. 
the correlation coefficients were lower than the coefficients using three growth stage combinations (data not shown).

The water-based NIR indices (WI and NWI) always provided higher genetic correlations with grain yield compared to the widely used indices (RNDVI, GNDVI, and SR) (Fig. 2). These patterns were also observed when individual growth stages and individual year/location yield were considered (data not shown). In most cases, the performance of the minor water band-based indices (WI and NWI) were indistinguishable from each other, but considering the overall performance, NWI-3 and NWI-4 showed better correlations with grain yield in the different experiments in different years and locations.

\section{Functional Relationship between Spectral Reflectance Indices and Grain Yield}

The functional relationship between grain yield and the different SRI for the three growth stages individually and in combination were established through regression analysis. Figure 3 shows the functional relationship of NWI-3 with yield in three growth stages for two experiments. The NWI-3 explained more of the grain yield variability at heading and grain-filling stages compared to the booting stage (Fig. 3). The relationship of three different indices (NWI-2, NWI-3, and NWI-4) with grain yield are presented in Fig. 4 for three different experiments, based on the mean of the three different growth stages in an

Table 7. Correlation coefficients between spectral reflectance indices and grain yield at three growth stages (GS) for three experiments.

\begin{tabular}{|c|c|c|c|c|c|c|c|}
\hline \multirow[t]{2}{*}{ Indices } & \multirow[t]{2}{*}{$\mathrm{GS}^{\dagger}$} & \multicolumn{2}{|c|}{ EXP-1 } & \multicolumn{2}{|c|}{ EXP-2 } & \multicolumn{2}{|c|}{ EXP-3 } \\
\hline & & STW 03/04 & LCB 03/04 & LCB 03/04 & LCB 04/05 & STW 03/04 & STW 04/05 \\
\hline \multirow[t]{4}{*}{ WI } & Boot & -0.374 & -0.272 & -0.105 & -0.358 & $-0.588^{\star \star}$ & -0.139 \\
\hline & $\mathrm{Hd}$ & $-0.674^{\star \star}$ & $-0.733^{\star \star}$ & $-0.643^{\star \star}$ & $-0.512^{\star \star}$ & $-0.686^{\star \star}$ & $-0.569^{\star \star}$ \\
\hline & GF & $-0.516^{\star \star}$ & $-0.672^{\star \star}$ & $-0.657^{\star \star}$ & $-0.752^{\star \star}$ & $-0.571^{\star \star}$ & $-0.685^{\star \star}$ \\
\hline & Mean $^{\ddagger}$ & $-0.814^{\star \star}$ & $-0.763^{\star \star}$ & $-0.676^{\star \star}$ & $-0.653^{\star \star}$ & $-0.785^{\star \star}$ & $-0.623^{\star *}$ \\
\hline \multirow[t]{4}{*}{ RNDVI } & Boot & 0.081 & 0.266 & 0.029 & 0.372 & 0.319 & 0.402 \\
\hline & $\mathrm{Hd}$ & $0.401^{*}$ & $0.481^{*}$ & $0.499^{*}$ & $0.400^{\star}$ & 0.259 & $0.525^{\star \star}$ \\
\hline & GF & 0.129 & $0.474^{\star}$ & $0.567^{\star \star}$ & $0.522^{\star \star}$ & 0.150 & $0.591^{\star \star}$ \\
\hline & Mean ${ }^{\ddagger}$ & 0.183 & $0.567^{\star \star}$ & $0.570^{\star \star}$ & $0.574^{\star *}$ & 0.232 & $0.605^{\star \star}$ \\
\hline \multirow[t]{4}{*}{ GNDVI } & Boot & 0.130 & 0.295 & 0.210 & $0.473^{*}$ & $0.397^{\star}$ & $0.469^{*}$ \\
\hline & $\mathrm{Hd}$ & $0.622^{\star \star}$ & $0.557^{\star *}$ & $0.680^{\star *}$ & $0.475^{\star}$ & $0.494^{\star}$ & $0.509^{\star \star}$ \\
\hline & GF & 0.108 & 0.387 & $0.512^{\star \star}$ & $0.478^{*}$ & 0.058 & $0.567^{\star \star}$ \\
\hline & Mean ${ }^{\ddagger}$ & 0.312 & $0.524^{\star \star}$ & $0.624^{\star \star}$ & $0.559^{\star \star}$ & 0.341 & $0.571^{\star \star}$ \\
\hline \multirow[t]{4}{*}{$\mathrm{SR}$} & Boot & 0.107 & 0.312 & -0.112 & 0.348 & 0.313 & $0.484^{\star}$ \\
\hline & $\mathrm{Hd}$ & $0.445^{\star}$ & $0.554^{\star \star}$ & $0.604^{\star \star}$ & $0.464^{*}$ & 0.367 & $0.594^{\star \star}$ \\
\hline & GF & 0.052 & $0.535^{\star \star}$ & $0.606^{\star \star}$ & $0.533^{\star *}$ & 0.224 & $0.608^{* \star}$ \\
\hline & Mean ${ }^{\ddagger}$ & 0.381 & $0.618^{\star \star}$ & $0.471^{\star}$ & $0.533^{\star *}$ & $0.435^{\star}$ & $0.595^{\star \star}$ \\
\hline \multirow[t]{4}{*}{ NWI-1 } & Boot & -0.364 & -0.268 & -0.097 & -0.366 & $-0.579^{\star \star}$ & -0.135 \\
\hline & $\mathrm{Hd}$ & $-0.673^{\star \star}$ & $-0.730^{\star \star}$ & $-0.650^{\star \star}$ & $-0.515^{\star \star}$ & $-0.682^{\star \star}$ & $-0.600^{\star \star}$ \\
\hline & GF & $-0.518^{\star \star}$ & $-0.663^{\star \star}$ & $-0.662^{\star \star}$ & $-0.750^{\star \star}$ & $-0.577^{\star \star}$ & $-0.684^{\star \star}$ \\
\hline & Mean ${ }^{\ddagger}$ & $-0.819^{\star \star}$ & $-0.758^{\star \star}$ & $-0.671^{\star \star}$ & $-0.653^{\star \star}$ & $-0.788^{\star \star}$ & $-0.618^{\star \star}$ \\
\hline \multirow[t]{4}{*}{ NWI-2 } & Boot & $-0.429^{\star}$ & -0.278 & -0.069 & -0.334 & $-0.598^{\star \star}$ & -0.172 \\
\hline & $\mathrm{Hd}$ & $-0.668^{\star \star}$ & $-0.690^{\star *}$ & $-0.617^{\star \star}$ & $-0.522^{\star \star}$ & $-0.650^{\star \star}$ & $-0.614^{\star \star}$ \\
\hline & GF & $-0.442^{\star}$ & $-0.596^{\star \star}$ & $-0.652^{\star \star}$ & $-0.645^{\star \star}$ & $-0.511^{\star \star}$ & $-0.670^{\star *}$ \\
\hline & Mean ${ }^{\ddagger}$ & $-0.759^{\star \star}$ & $-0.702^{\star \star}$ & $-0.690^{\star *}$ & $-0.657^{\star \star}$ & $-0.741^{\star \star}$ & $-0.622^{\star \star}$ \\
\hline \multirow[t]{4}{*}{ NWI-3 } & Boot & $-0.423^{*}$ & -0.260 & -0.106 & -0.348 & $-0.560^{\star \star}$ & -0.132 \\
\hline & $\mathrm{Hd}$ & $-0.704^{\star \star}$ & $-0.740^{\star \star}$ & $-0.678^{\star \star}$ & $-0.522^{\star \star}$ & $-0.666^{\star \star}$ & $-0.581^{\star *}$ \\
\hline & GF & $-0.514^{\star \star}$ & $-0.703^{\star \star}$ & $-0.742^{\star \star}$ & $-0.729^{\star \star}$ & $-0.579^{\star \star}$ & $-0.698^{\star \star}$ \\
\hline & Mean ${ }^{\ddagger}$ & $-0.865^{\star \star}$ & $-0.798^{\star \star}$ & $-0.776^{\star \star}$ & $-0.666^{\star \star}$ & $-0.777^{\star \star}$ & $-0.668^{\star \star}$ \\
\hline \multirow[t]{4}{*}{ NWI-4 } & Boot & $-0.499^{\star}$ & -0.281 & -0.089 & -0.335 & $-0.581^{\star \star}$ & -0.164 \\
\hline & $\mathrm{Hd}$ & $-0.707^{\star \star}$ & $-0.727^{\star \star}$ & $-0.649^{\star *}$ & $-0.516^{\star \star}$ & $-0.658^{\star \star}$ & $-0.598^{\star \star}$ \\
\hline & GF & $-0.478^{\star}$ & $-0.646^{\star \star}$ & $-0.721^{\star *}$ & $-0.670^{\star *}$ & $-0.551^{\star *}$ & $-0.689^{\star \star}$ \\
\hline & Mean ${ }^{\ddagger}$ & $-0.845^{\star \star}$ & $-0.810^{\star \star}$ & $-0.753^{\star \star}$ & $-0.673^{\star \star}$ & $-0.761^{\star \star}$ & $-0.684^{\star \star}$ \\
\hline
\end{tabular}

*Significant at the 0.05 probability level.

${ }^{*}$ Significant at the 0.01 probability level.

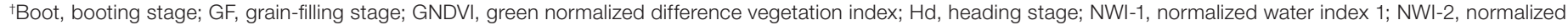
water index 2; NWI-3, normalized water index 3; NWI-4, normalized water index 4; RNDVI, red normalized difference vegetation index; SR, simple ratio; WI, water index.

${ }^{\ddagger}$ Correlation between yield and mean spectral reflectance indices across three growth stages. 

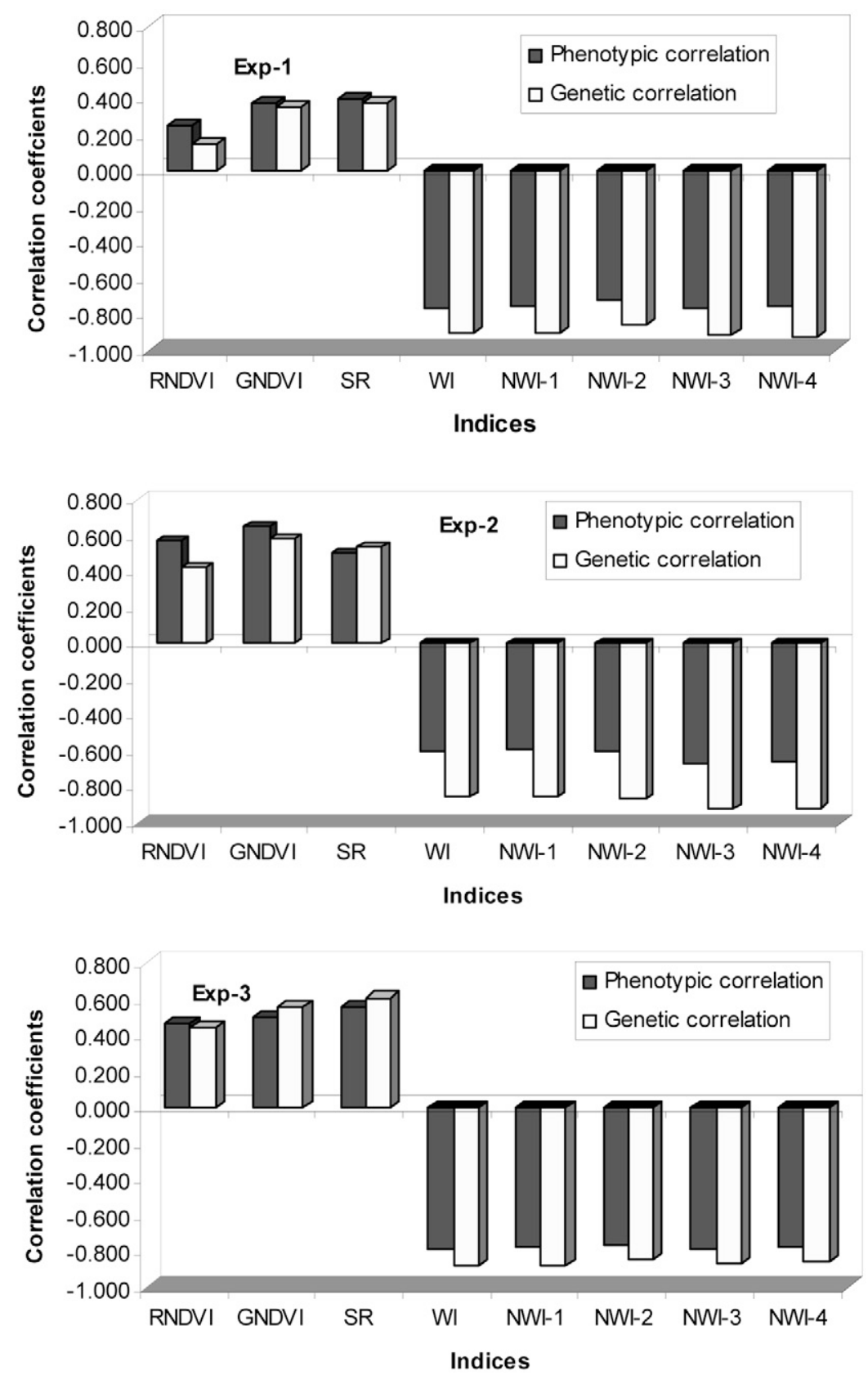

Figure 2. Phenotypic and genetic correlation coefficients between different spectral reflectance indices (mean of different growth stages across year/ location) and grain yield (mean of two locations in Exp-1 and 2 yr in Exp2 and Exp-3). GNDVI, green normalized difference vegetation index; NWI-1, normalized water index 1; NWI-2, normalized water index 2; NWI-3, normalized water index 3; NWI-4, normalized water index 4; RNDVI, red normalized difference vegetation index; SR, simple ratio; WI, water index.

individual year or location. The relationships of the other indices with grain yield were also tested (data not shown), and in all instances the model fit better with a linear relationship having the higher coefficient of determination compared to other models. Indices tended to explain more of the grain yield variability when mean data were used, for example, combining growth stages, combining years/ locations, or combining both. Our analysis based on combining growth stages revealed the superiority of NWI-3 over the other indices in predicting yield, explaining $74 \%$ of the grain yield variation in one location and $63 \%$ of the variation in the other location for Exp-1 (Fig. 4). The relationship of measured grain yield and predicted grain yield based on NWI-3 is presented in Fig. 5. This relationship was established on the basis of all three experiments using the mean values of the three growth stages across locations and years. Although some differences were observed between the different experiments for grain yield and spectral behavior, the relationships revealed the significant linear predictability of grain yield based on NWI-3.

\section{Selection of Genotypes}

Significant difference between genotypic grain yields of the two selection groups based on the $25 \%$ highest and the 25\% lowest SRI values was observed for all the water-based SRI (data not shown). The water index and the NWI showed greater accuracy in identifying both the highestyielding and the lowest-yielding genotypes compared to the RNDVI, GNDVI, and SR (Table 8). Similar trends were also observed when individual growth stages were considered (data not shown), but the combination of growth stages always worked better. The indices that included the minor water absorption band identified 50 to $83 \%$ of both the $25 \%$ highest-yielding genotypes and the 25\% lowest-yielding genotypes in all three experiments. Among the NIR-based indices, NWI-3 and NWI-4 consistently performed better. This was also evident when the mean of the two locations or 2-yr data were combined for individual experiments, and also when the three experiments were considered together (Table 8).

Yield per se and yield estimates of the 25\% highest-yielding and 25\% lowest-yielding genotypes for the three experiments based on the linear regression equation of the different SRI are presented in Table 9. The percent difference between the harvested and predicted yield in different experiments was as close as $0.9 \%$ for the highest-yielding genotypes using NWI-3 in Exp-1. The largest difference between actual and predicted yield was $21.1 \%$ for the RNDVI in Exp-3. In most cases, the percent yield difference was $10 \%$ or less, with the water-based NIR indices commonly having differences of 1 to $5 \%$. The widely used indices (RNDVI, GNDVI, and SR) showed inconsistency across years and locations in identifying the highest-yielding and lowest-yielding genotypes, while the NIR indices showed remarkable accuracy and consistency in predicting the relative performance of the genotypes for grain yield across years and locations. 

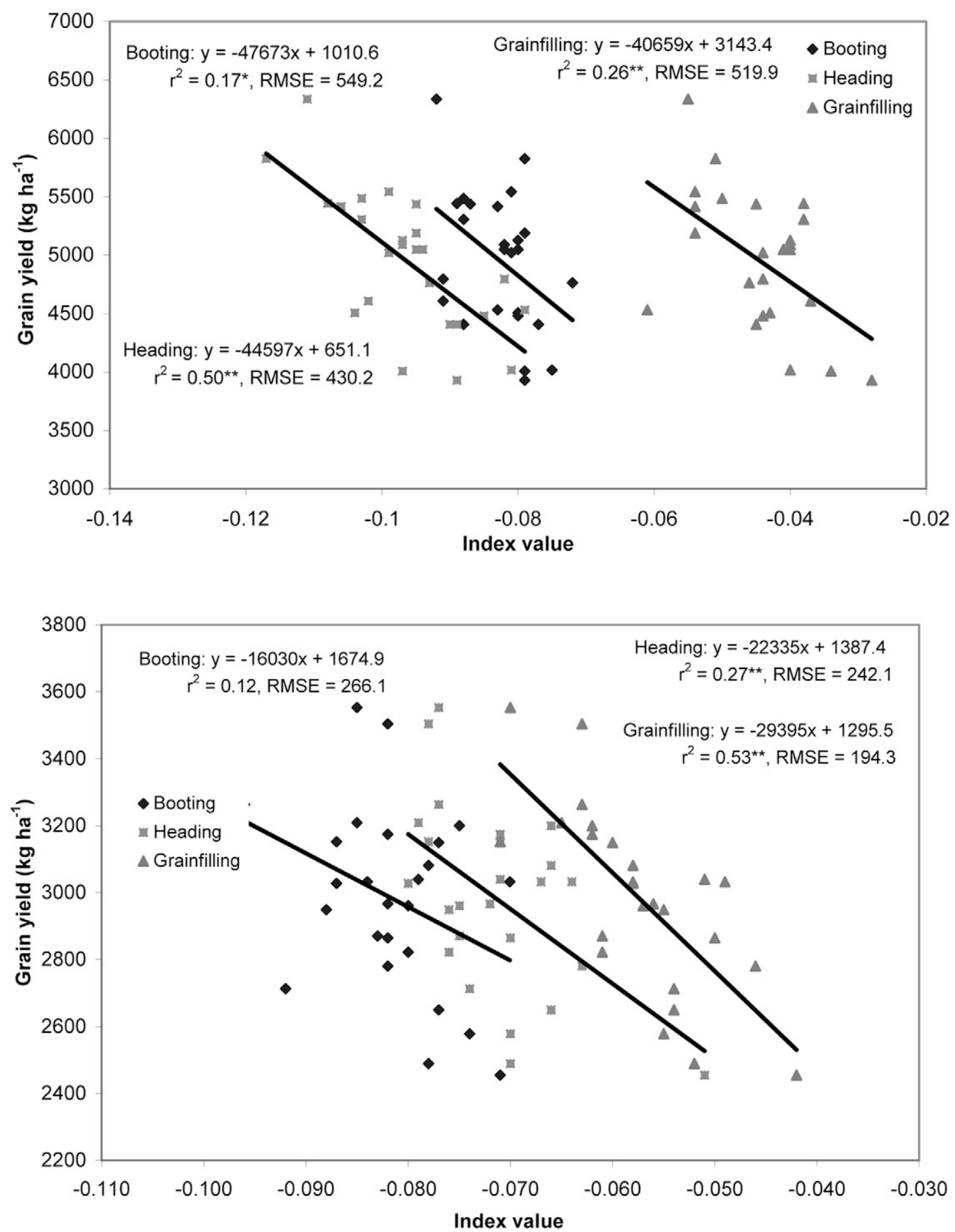

Figure 3. Functional relationship between grain yield and normalized water index 3 (NWI-3) at three growth stages for two experiments. Top: Exp-1; bottom: Exp-2. *Significant at the 0.05 probability level; ${ }^{* *}$ significant at the 0.01 probability level. RMSE, root mean square error.

\section{DISCUSSION}

\section{Genotypic Variation and Growth Stage}

Significant genotypic variation for grain yield and spectral behavior at different growth stages, namely booting, heading, and grain-filling, confirms the existence of sufficient variation among the genotypes. The first experiment (Exp-1) includes widely used winter wheat cultivars available throughout the Great Plains of the USA. The other two experiments contain sufficient variation in the respective RIL populations. Similar variations were also observed by other researchers working with spectral reflectance in wheat, including Babar et al. (2006) working with irrigated spring wheat, with irrigated and nonirrigated spring wheat (Gutiérrez-Rodríguez et al., 2004), with durum wheat in the Mediterranean region (Aparicio et al., 2000, 2002), and with durum wheat under rainfed conditions (Royo et al., 2003).The primary reason for the decreasing trend of RNDVI, GNDVI, and SR from booting to grain-filling is the reduced reflectance in the NIR region due to loss of green tissue as growth 

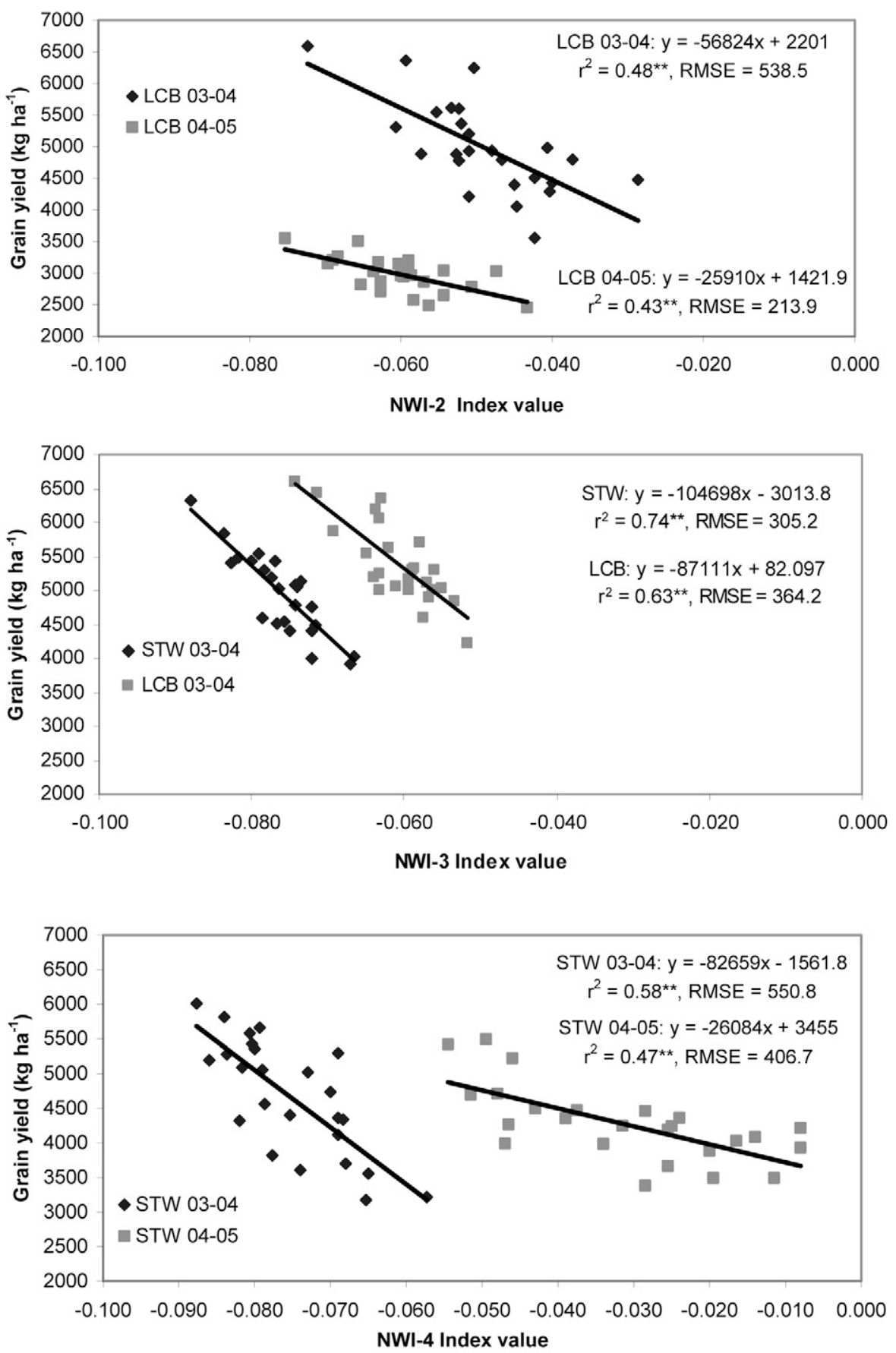

Figure 4. Functional relationship between grain yield and normalized water index 2 (NWI-2), normalized water index 3 (NWI-3), and normalized water index 4 (NWI-4) (estimated as the mean of the three growth stages). Top: Exp-2; middle: Exp-1; bottom: Exp-3. STW and LCB denote Stillwater and Lake Carl Blackwell sites, respectively. ${ }^{* *}$ Significant at the 0.01 probability level. RMSE, root mean square error.

stage progressed from booting to grain-filling, as well as increased reflectance in the visible region (Aparicio et al., 2000). The NIR indices (WI and NWI) are based on the minor water absorption band $(970 \mathrm{~nm})$, and they measure the water status of the canopy (Peñuelas et al., 1997; Babar et al., 2006). In 2003/2004, the experiments had good water status during heading (71.4-74.9 $\mathrm{mm}$ rainfall) and the indices were lower than at booting stage, whereas, in $2004 / 2005$, heading occurred under water deficit stress conditions (9.9-10.7 $\mathrm{mm}$ rainfall) and the indices were higher at heading compared to booting stage (Fig. 1), since there is a negative relationship between water status and indices values. The nonsignificant difference among the genotypes for the SRI in some instances at the booting stage was due to the homogeneity of the LAI among the genotypes, which normally reach maximum at the booting stage (Aparicio et al., 2000). But, at the later growth stages, these indices are highly influenced by the reproductive structures such as spike morphology, as well as the decrease in the LAI, and indices detected the significant variation among the genotypes (Hatfield, 1981; Aparicio et al., 2000). The commonly used indices (NDVI and SR) usually saturate at LAI $>3$, which is due to the lack of sensitivity of red light at the higher vegetation fractions. Whereas, the NIR bands of the water-based indices (WI and NWI) can penetrate into the higher vegetation fraction of the canopy and assess the water status of the canopy as well as the amount of green biomass (Babar et al., 2006).

\section{Interaction Effect of Genotypes, Growth Stages, and Years/Locations}

We observed significant interaction between different growth stages and the genotypes for different indices (Table 5). This means the specific growth stages are a very important consideration when measuring the spectral reflectance. Similar observations were made in the studies of Babar et al. (2006) with irrigated spring wheat and by Aparicio et al. (2002) with rain-fed durum wheat. We also observed very low associations for any individual index between different growth stages, especially between the booting and the heading stages, and between the booting and the grain-filling stages, with a few exceptions. This indicates genotypes were ranked differently for indices value in different growth stages, which is similar to observations reported by Babar et al. (2006) and Royo et al. (2003). Correlations between the heading and grain-filling stages of individual indices were significant for all cases except Exp-1 at Stillwater in 2003/2004. Since heading and grain-filling stages are the two most important growth stages for spectral reflectance measurements, we recommend measuring spectral reflectance at these two growth stages. 
Correlation between Spectral Reflectance Indices and Grain Yield

Different studies have reported the usefulness of spectral reflectance indices for predicting grain yield under different environmental conditions. Most studies considered the diversity of the environments in differentiating genotypes, including different water regimes in durum wheat (Aparicio et al., 2000), salinity effects in barley (Peñuelas et al., 1997), fertilizer and water treatments in corn (Osborne et relationships between WI and relative water content, leaf water potential, stomatal conductance, and canopy temperature. The minor water absorption band $(970 \mathrm{~nm})$ and other NIR bands used in the calculation of WI and NWI make these indices more sensitive to higher fractions of green vegetation at the heading and grain-filling growth stages compared to NDVI and SR (Babar et al., 2006). The higher correlation coefficients of the NIR-based indices with genotypic grain yield compared to NDVI and SR are al., 2002), and plant population density in soybean (Ma et al., 2001). These studies measured the environmental influence in increasing the variability in the experiments rather than the genetic variability for grain yield due to genotypes. However, Babar et al. (2006) established a strong relationship between NIR-based indices and grain yield with irrigated spring wheat genotypes. In contrast, there has been little research in using spectral reflectance indices in predicting winter wheat genotypes for grain yield under Great Plains rain-fed conditions. Raun et al. (2001) previously reported the use of NDVI in predicting the yield of a few winter wheat cultivars under diverse nitrogen levels.

We used six published SRI (RNDVI, GNDVI, SR, WI, NWI-1, and NWI-2), and two new indices (NWI-3 and NWI4) were calculated (Table 2). Most indices taken at the booting stage showed nonsignificant correlations with grain yield in the different experiments, with a few exceptions. The relationship between SRI and yield became stronger as the growth stage progressed toward the reproductive phase. Babar et al. (2006) also reported lower correlations between the different spectral reflectance indices and grain yield at the booting stage. Ma et al. (2001) with soybean and Royo et al. (2003) with durum wheat also reported that the SRI correlated better with yield at the reproductive growth stages rather than at early vegetative growth stages. Aparicio et al. (2000) found significant correlations between grain yield and SRI at hard dough stage (Zadoks stage 87) with durum wheat under rain-fed conditions.

Water index and the NWI gave higher correlations with grain yield compared with the RNDVI, GNDVI, and SR at heading and grain-filling (Table 7). Peñuelas et al. (1993) reported significant
Table 8 . The percentage of $25 \%$ highest-yielding and $25 \%$ lowest-yielding (values in parentheses) genotypes selected by different spectral reflectance indices.

\begin{tabular}{lccccccccc}
\hline \multirow{2}{*}{ Experiments } & \multicolumn{8}{c}{ Spectral reflectance indices $^{\dagger}$} \\
& WI & RNDVI & GNDVI & SR & NWI-1 & NWI-2 & NWI-3 & NWI-4 \\
\hline EXP-1 & & & & & & & & \\
STW 03/04 & $83(83)$ & $17(33)$ & $50(33)$ & $50(50)$ & $83(67)$ & $67(67)$ & $83(83)$ & $83(67)$ \\
LCB 03/04 & $83(50)$ & $50(50)$ & $50(50)$ & $50(67)$ & $67(50)$ & $83(50)$ & $67(83)$ & $83(67)$ \\
Mean & $67(50)$ & $50(33)$ & $50(50)$ & $50(33)$ & $67(50)$ & $67(50)$ & $83(83)$ & $83(83)$ \\
EXP-2 & & & & & & & & \\
LCB 03/04 & $67(50)$ & $67(50)$ & $67(50)$ & $67(50)$ & $67(50)$ & $67(50)$ & $83(67)$ & $83(67)$ \\
LCB 04/05 & $67(50)$ & $67(50)$ & $50(50)$ & $50(50)$ & $50(50)$ & $67(50)$ & $83(67)$ & $83(67)$ \\
Mean ${ }^{\ddagger}$ & $50(50)$ & $50(33)$ & $33(67)$ & $50(50)$ & $50(50)$ & $67(33)$ & $67(67)$ & $67(67)$ \\
EXP-3 & & & & & & & & \\
STW 03/04 & $50(67)$ & $33(17)$ & $33(33)$ & $50(50)$ & $50(67)$ & $67(50)$ & $67(67)$ & $67(67)$ \\
STW 04/05 & $67(50)$ & $50(50)$ & $50(50)$ & $83(50)$ & $67(50)$ & $83(50)$ & $83(50)$ & $83(67)$ \\
Mean ${ }^{\ddagger}$ & $67(50)$ & $67(17)$ & $83(33)$ & $83(33)$ & $67(67)$ & $67(67)$ & $83(83)$ & $83(83)$ \\
Overall mean $^{\S}$ & $61(50)$ & $56(28)$ & $55(50)$ & $61(39)$ & $61(56)$ & $67(50)$ & $78(78)$ & $78(78)$ \\
\hline
\end{tabular}

${ }^{+}$GNDVI, green normalized difference vegetation index; NWI-1, normalized water index 1; NWI-2, normalized water index 2; NWI-3, normalized water index 3; NWI-4, normalized water index 4; RNDVI, red normalized difference vegetation index; SR, simple ratio; WI, water index.

¥Selection based on mean spectral reflectance indices across three growth stages and mean grain yield across locations/years for each individual experiment.

sSelection based on mean spectral reflectance indices across three growth stages and mean grain yield across locations/years for all three experiments.

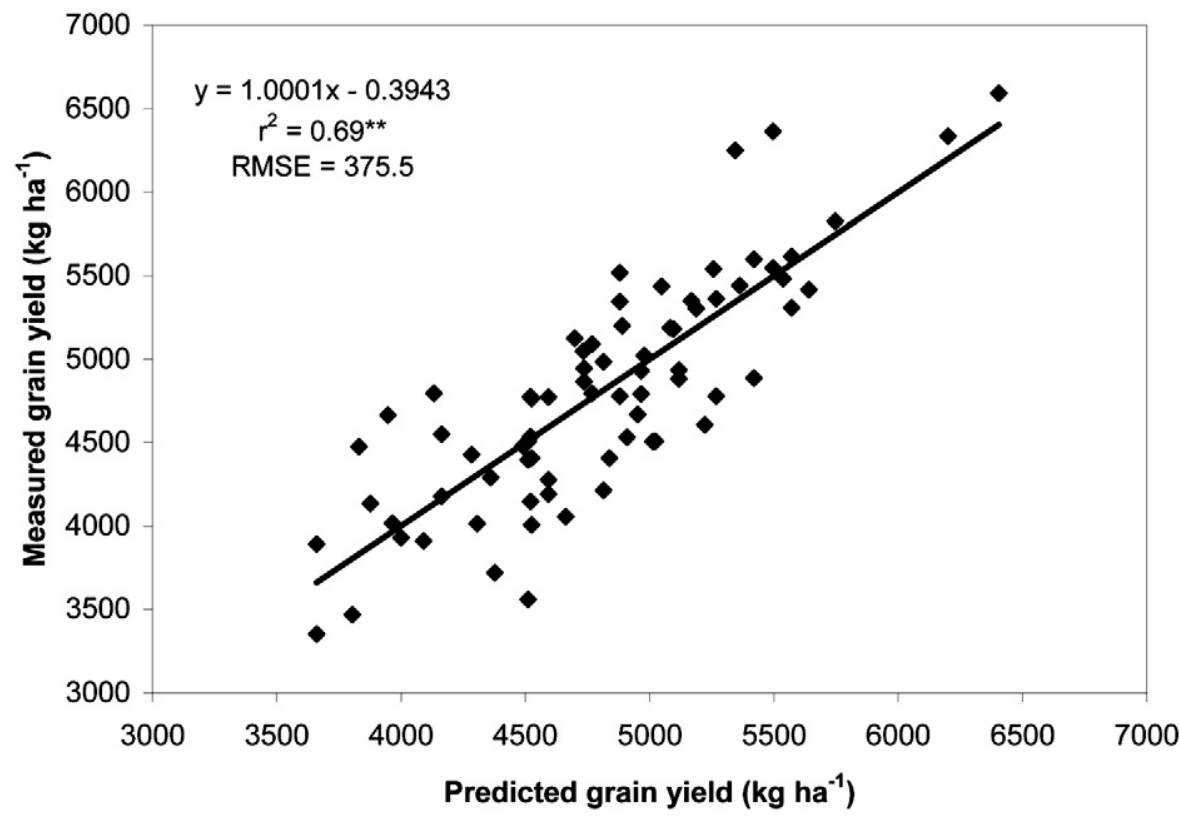

Figure 5. Relationship between measured grain yield and predicted grain yield based on the linear equation using normalized water index 3 (NWI-3) as the predictor, estimated using the mean values of three growth stages for all three experiments across two locations/years. **Significant at the 0.01 probability level. RMSE, root mean square error. 
Table 9. Comparison between measured grain yield $\left(\mathrm{kg} \mathrm{ha}^{-1}\right)$ and predicted grain yield $\left(\mathrm{kg} \mathrm{ha}^{-1}\right)$ of the $25 \%$ highest-yielding and $25 \%$ lowest-yielding (values in parentheses) genotypes in each experiment using spectral reflectance indices.

\begin{tabular}{|c|c|c|c|c|c|c|c|c|}
\hline \multirow[t]{2}{*}{ Yield per se ${ }^{\dagger}$} & \multicolumn{8}{|c|}{ Predicted grain yield by spectral reflectance indices ${ }^{\ddagger}$} \\
\hline & WI & RNDVI & GNDVI & SR & NWI-1 & NWI-2 & NWI-3 & NWI-4 \\
\hline \multicolumn{9}{|l|}{ EXP-1 } \\
\hline $\begin{array}{l}\text { STW 03/04 } \\
5678(4207)\end{array}$ & $5620(4386)$ & $5071(4797)$ & $5170(4963)$ & $5228(4677)$ & $5624(4380)$ & $5595(4379)$ & $5627(4333)$ & $5622(4352)$ \\
\hline Difference ${ }^{\S}$ & $1.0(4.2)$ & $10.7(14.0)$ & $9.0(11.6)$ & $7.9(11.1)$ & $1.0(4.1)$ & $1.5(4.1)$ & $0.9(3.0)$ & $1.0(3.4)$ \\
\hline $\begin{array}{l}\text { LCB 03/04 } \\
6248 \text { (4758) }\end{array}$ & $6006(4837)$ & $5852(5029)$ & $5764(4983)$ & $5852(4994)$ & $5993(4845)$ & $6001(4865)$ & 6000 (4857) & 6005 (4843) \\
\hline Difference & $3.9(1.7)$ & $6.3(5.7)$ & $7.7(4.7)$ & $6.3(5.0)$ & $4.1(1.8)$ & $4.0(2.2)$ & $4.0(2.1)$ & $3.9(1.8)$ \\
\hline \multicolumn{9}{|l|}{ EXP-2 } \\
\hline $\begin{array}{l}\text { LCB 03/04 } \\
5995 \text { (4157) }\end{array}$ & $5621(4337)$ & 5504 (4455) & $5573(4383)$ & 5567 (4439) & $5628(4327)$ & 5585 (4358) & 5662 (4280) & 5631 (4302) \\
\hline Difference & $6.2(4.3)$ & $8.2(7.2)$ & $7.0(5.4)$ & $7.1(6.8)$ & $6.1(4.1)$ & $6.8(4.8)$ & $5.6(3.0)$ & $6.1(3.5)$ \\
\hline $\begin{array}{l}\text { LCB 04/05 } \\
3317 \text { (2611) }\end{array}$ & 3205 (2725) & 3147 (2765) & 3139 (2770) & 3177 (2753) & 3211 (2728) & 3208 (2739) & 3197 (2744) & 3203 (2739) \\
\hline Difference & $3.4(4.4)$ & $5.1(5.9)$ & $5.4(6.1)$ & $4.2(5.4)$ & $3.2(4.5)$ & $3.3(4.9)$ & $3.6(5.1)$ & $3.4(4.9)$ \\
\hline \multicolumn{9}{|l|}{ EXP-3 } \\
\hline $\begin{array}{l}\text { STW 03/04 } \\
5642 \text { (3511) }\end{array}$ & 5407 (3809) & 4931 (4450) & 5042 (4324) & 5157 (4272) & 5410 (3804) & 5388 (3873) & 5402 (3791) & 5396 (3853) \\
\hline Difference & $4.2(7.8)$ & $12.6(21.1)$ & 10.6 (18.8) & $8.6(17.8)$ & $4.1(7.7)$ & $4.5(9.3)$ & $4.2(7.4)$ & $4.3(8.8)$ \\
\hline $\begin{array}{l}\text { STW 04/05 } \\
5008 \text { (3640) }\end{array}$ & 4728 (3800) & 4686 (3857) & 4653 (3844) & 4758 (3864) & 4728 (3802) & 4742 (3799) & 4723 (3807) & 4747 (3802) \\
\hline Difference & $5.6(4.4)$ & $6.4(5.9)$ & $7.1(5.6)$ & $5.0(6.2)$ & $5.6(4.4)$ & $5.3(4.4)$ & $5.7(4.6)$ & $5.2(4.4)$ \\
\hline
\end{tabular}

tSTW, Stillwater site; LCB, Lake Carl Blackwell site.

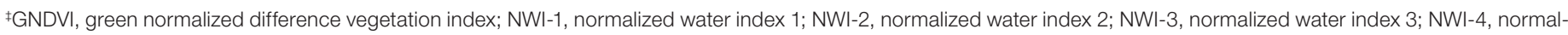
ized water index 4; RNDVI, red normalized difference vegetation index; SR, simple ratio; WI, water index.

§Difference between measured grain yield and predicted grain yield, expressed as percentage.

due to better differentiation of the genotypes at heading and grain-filling. It has been also reported that WI can predict relative water content, stomatal conductance, and leaf water potential (Peñuelas et al., 1993). The correlation coefficients of different water-based indices with grain yield were different but negligible, which was due to the use of different NIR wavebands to normalize the 970 -nm water band in different NIR indices. Increased water content in the plant canopy decreased the reflectance of the water band, and consequently, we obtained negative correlations of these indices with grain yield. Babar et al. (2006) reported similar observations.

Correlations of the mean estimates of the SRI across three growth stages with yield were higher compared to any individual growth stage (Table 7). This was also reported by Babar et al. (2006) and Aparicio et al. (2000). The NIR-based indices always gave higher correlations than the RNDVI, GNDVI, and SR when reflectance measurements were combined across different growth stages. In most cases, the mean of the indices at the heading and the grain-filling stages also gave higher correlations with grain yield than any individual growth stage, but lower than the correlation coefficients obtained through combining three growth stages (data not shown). We believe the mean indices across growth stages are a measurement of overall plant health during a critical time in plant development. Repeated reflectance measurements of a genotype from different growth stages assess the overall fitness of the genotype with time. Therefore, the indices identify the genotypes that maintain a healthier condition throughout the growing season and, as a result, give higher correlations with final grain yield. We observed higher genetic correlations between the water-based NIR indices and grain yield compared to the widely used vegetation based indices (Fig. 2). This observation indicated that these water-based indices have higher predictability at the genotypic level for grain yield variation compared to the vegetation based indices under rain-fed conditions.

\section{Functional Relationship between the SRI and Grain Yield}

The functional relationship between the SRI and grain yield was linear (Figs. 3 and 4). Babar et al. (2006) and Gutiérrez-Rodríguez et al. (2004) also reported linear relationships between the different SRI and grain yield. Our results indicate that these SRI can be used for predicting grain yield irrespective of the yield potential of the genotypes. The NIR-based indices always showed better predictability when more data were included in the model (different growth stages, different years/locations). These indices also predicted grain yield well when an individual growth stage such as heading or grain-filling stages was 
considered, thus making them suitable as a selection tool in a breeding program for yield improvement. Considering the overall performance, NWI-3 and NWI-4 showed better performance over the other NIR-based indices.

\section{Selection of Genotypes}

The efficiency of selecting superior genotypes is a primary concern when using an indirect selection tool for a specific trait. Significant yield differences between the two groups of genotypes based on the highest and lowest index values for the water-based indices indicate that these indices can properly discriminate the genotypes for grain yield. Our results showed that the water-based NIR indices identified a major proportion of the higher-yielding as well as the lower-yielding genotypes in the different experiments. In addition, the close approximation of actual grain yield reveals the power of the indices for prediction of the genotypes grain yield. Use of the NIR-based indices, especially NWI-3 and NWI-4, shows promise in selecting desired genotypes for higher grain yield.

\section{CONCLUSIONS}

The potential of SRI for predicting winter wheat genotypes for grain yield variability is demonstrated in our study. The randomly derived recombinant inbred lines established the genetic basis of the relationship between the SRI and grain yield. The indices were related to grain yield in a linear fashion confirming the effectiveness for identifying the higheryielding genotypes irrespective of their yield potential. Combining the reflectance measurements from three growth stages provided the best relationship between SRI and grain yield, but for practical consideration, we recommend taking spectral reflectance measurements at the heading and the grain-filling stages for predicting the genotypic grain yield potential. Indices based on the minor water absorption band consistently provided the best relationships with grain yield, and among them NWI-3 and NWI-4 showed better performance in identifying the higher-yielding genotypes in different winter wheat genetic backgrounds in the Great Plains.

\section{References}

Aparicio, N., D. Villegas, J.L. Araus, J. Casadesus, and C. Royo. 2002. Relationship between growth traits and spectral vegetation indices in durum wheat. Crop Sci. 42:1547-1555.

Aparicio, N., D. Villegas, J. Casadesus, J.L. Araus, and C. Royo. 2000. Spectral vegetation indices as nondestructive tools for determining durum wheat yield. Agron. J. 92:83-91.

Araus, J.L., J. Casadesus, and J. Bort. 2001. Recent tools for the screening of physiological traits determining yield. p. 59-77. In M.P. Reynolds et al. (ed.) Application of physiology in wheat breeding. CIMMYT, Mexico, DF.

Araus, J.L., G.A. Slafer, M.P. Reynolds, and C. Royo. 2002. Plant breeding and drought in $\mathrm{C}_{3}$ cereals: What should we breed for? Ann. Bot. (Lond.) 89:925-940.

Babar, M.A., M.P. Reynolds, M. van Ginkel, A.R. Klatt, W.R. Raun, and M.L. Stone. 2006. Spectral reflectance indices as a potential indirect selection criteria for wheat yield under irrigation. Crop Sci. 46:578-588.

Ball, S.T., and C. Konzak. 1993. Relationship between grain yield and remotely sensed data in wheat breeding experiments. Plant Breed. 110:277-282.

Baret, F., and G. Guyot. 1991. Potentials and limits of vegetation indices for LAI and APAR estimation. Remote Sens. Environ. 35:161-173.

Falconer, D.S. 1989. Introduction to quantitative genetics. 3rd ed. Longman Scientific and Technical, New York.

Gitelson, A.A., Y.J. Kaufman, and M.N. Merzylak. 1996. Use of a green channel in remote sensing of global vegetation from EOS-MODIS. Remote Sens. Environ. 58:289-298.

Gutiérrez-Rodríguez, M., M.P. Reynolds, J.A. Escalente-Estrada, and M.T. Rodríguez-González. 2004. Association between canopy reflectance indices and yield and physiological traits in bread wheat under drought and well-irrigated conditions. Aust. J. Agric. Res. 55:1139-1147.

Hatfield, J.L. 1981. Spectral behavior of wheat yield variety trials. Photogramm. Eng. Remote Sens. 47:1487-1491.

Jackson, P., M. Robertson, M. Copper, and G. Hammer. 1996. The role of physiological understanding in plant breeding, from a breeding perspective. Field Crops Res. 49:11-37.

Knipling, E.B. 1970. Physical and physiological basis for the reflectance of visible and near-infrared radiation from vegetation. Remote Sens. Environ. 1:155-159.

Kobayashi, K., and M.U. Salam. 2000. Comparing simulated and measured values using mean squared deviation and its components. Agron. J. 92:345-350.

Ma, B.L., L.M. Dwyer, C. Costa, E.L. Cober, and M.J. Morrison. 2001. Early prediction of soybean yield from canopy reflectance measurements. Agron. J. 93:1227-1234.

Osborne, S.L., J.S. Schepers, D.D. Francis, and M.R. Schlemmer. 2002. Use of spectral radiance to estimate in-season biomass and grain yield in nitrogen and water-stressed corn. Crop Sci. 42:165-171.

Peñuelas, J., I. Filella, C. Biel, L. Serrano, and R. Save. 1993. The reflectance at the $950-970 \mathrm{~nm}$ region as an indicator of plant water status. Int. J. Remote Sens. 14:1887-1905.

Peñuelas, J., R. Isla, I. Filella, and J.L. Araus. 1997. Visible and near-infrared reflectance assessment of salinity effects on barley. Crop Sci. 37:198-202.

Price, J.C., and W.C. Bausch. 1995. Leaf area index estimation from visible and near-infrared reflectance data. Remote Sens. Environ. 52:55-65.

Raun, W.R., J.B. Solie, G.V. Johnson, M.L. Stone, E.V. Lukina, W.E. Thomason, and J.S. Schepers. 2001. In-season prediction of potential grain yield in winter wheat using canopy reflectance. Agron. J. 93:131-138.

Reynolds, M.P., S. Rajaram, and K.D. Sayre. 1999. Physiological and genetic changes of irrigated wheat in the post-green revolution period and approaches for meeting projected global demand. Crop Sci. 39:1611-1621.

Reynolds, M.P., R.M. Trethowan, M. van Ginkel, and S. Rajaram. 2001. Application of physiology in wheat breeding. p. 2-10. In M. P. Reynolds et al. (ed.) Application of physiology in wheat breeding. CIMMYT, Mexico, DF.

Richards, R.A. 1996. Defining selection criteria to improve yield under drought. Plant Growth Regul. 20:157-166.

Richards, R.A. 1982. Breeding and selecting for drought resistant wheat. p. 303-316. In Drought resistance in crops with emphasis on rice. IRRI, Manila, Philippines. 
Royo, C., N. Aparicio, D. Villegas, J. Casadesus, P. Monneveux, and J.L. Araus. 2003. Usefulness of spectral reflectance indices as durum wheat yield predictors under contrasting Mediterranean conditions. Int. J. Remote Sens. 24:4403-4419.

SAS Institute. 2001. The SAS system for windows, version 8.2. SAS Inst., Cary, NC.

Serrano, L., I. Filella, and J. Peñuelas. 2000. Remote sensing of biomass and yield of winter wheat under different nitrogen supplies. Crop Sci. 40:723-731.

Tucker, J.C., B.N. Holben, J.H. Elgin, Jr., and J.E. McMurtrey,
III. 1980. Relationship of spectral data to grain yield variation. Photogramm. Eng. Remote Sens. 46:657-666.

Wiegand, C.L., and A.J. Richardson. 1990a. Use of spectral vegetation indices to infer leaf area, evapotranspiration, and yield. I. Rationale. Agron. J. 82:623-629.

Wiegand, C.L., and A.J. Richardson. 1990b. Use of spectral vegetation indices to infer leaf area, evapotranspiration, and yield: II. Results. Agron. J. 82:630-636.

Zadoks, J.C., T.T. Chang, and C.F. Konzak. 1974. A decimal code for the growth stages of cereals. Weed Res. 14:415-421. 